\title{
The Mathematical Life of Pierre Leroux
}

\author{
Gilbert Labelle \\ * Département de mathématiques, Université du Québec à Montréal, Montréal (Québec) Canada H3C 3P8 \\ Email: labelle.gilbert@UQAM.ca \\ Received: April 1, 2021, Accepted: April 22, 2021, Published: April 30, 2021 \\ The authors: Released under the CC BY-ND license (International 4.0)
}

\begin{abstract}
The present "historical" paper describes the mathematical life of Pierre Leroux from my point of view as a colleague, main collaborator, and friend. It is an adaptation in English, slightly augmented and enriched with figures and photos, of my paper [38] which appeared in French in the Gazette des mathématiciens (Société mathématique de France) under the title La carrière mathématique de Pierre Leroux.
\end{abstract}

Keywords: Combinatorics; Discrete mathematics; Theory of combinatorial species

2020 Mathematics Subject Classification: 05A05; 05A15

\section{Introduction}

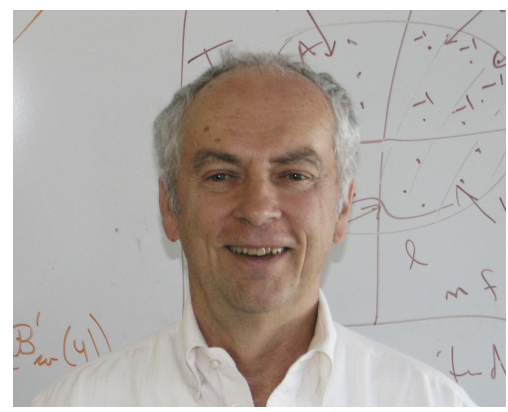

Professor Pierre Leroux UQÀM 2006.
Pierre Leroux (1942-2008) was a professor in the mathematics department of Université du Québec à Montréal (UQÀM) from June 1971 to August 2007, when he took his retirement. Far from ceasing his research and student supervision activities, he had taken them all over again until the moment of his completely unexpected death, on March 9, 2008.

\section{Early mathematical activities}

The early mathematical activities of Pierre Leroux began in the mid-sixties through his work in algebra and, more specifically, in category theory. At the Université de Montréal, in 1965, he published a master thesis [50] on the existence of injective envelopes then, in 1970 under the supervision of Jean-Marie Maranda, a remarkable doctoral thesis [51] on the extension of a pair of adjoint functors to categories of morphisms. In 1970-71 he worked as a post-doctoral research fellow of NSERC (Canada), at the Laboratoire de mathématiques, Faculté des sciences d'Orsay (France).

Then came his first three research articles published in international scientific journals: the first, in 1970 in collaboration with Paul Ribenboim, on higher order derivations in semi-additive categories [62], followed in 1971-72, by two publications [52,53] dealing respectively with erasure structures, and a characterization of the category of groups.

In 1975, he introduced an interesting extension, at the category level, of the Möbius inversion which was until then confined to number theory, and more generally, to the context of incidence algebras. He thus created a new class of categories he named Möbius categories [54]. From 1980 to 1982, in collaboration with Mireille Content, François Lemay and Jean Saraillé, he published three other articles on various properties of Möbius 
categories $[15,55,67]$. He also wrote, in 1985 with R.E Block, an article Generalized dual coalgebras of algebras, with applications to cofree coalgebras [7].

\section{He discovers the world of modern combinatorics}

During a sabbatical year at the mathematics department of the University of San Diego (UCSD), 1978-79, he discovers the world of modern combinatorics by collaborating with researchers including Adriano Garsia, Richard Stanley, Gérard Xavier Viennot, etc. His enthusiasm for this subject is such that he started in 1979, on his return to UQÀM, a new weekly research seminar: the Séminaire de Combinatoire. This seminar was so successful with the national and international mathematical community, that its activities, every Friday, still exist today, even after over forty years.

It is under the impetus of this seminar that the Groupe de Recherche en Combinatoire de l'UQÀM, was born around the 1980s, initially bringing together André Joyal, Gilbert Labelle, Jacques Labelle, Pierre Bouchard, François Bergeron, Hélène Décoste, etc. A multitude of other researchers having been added since.

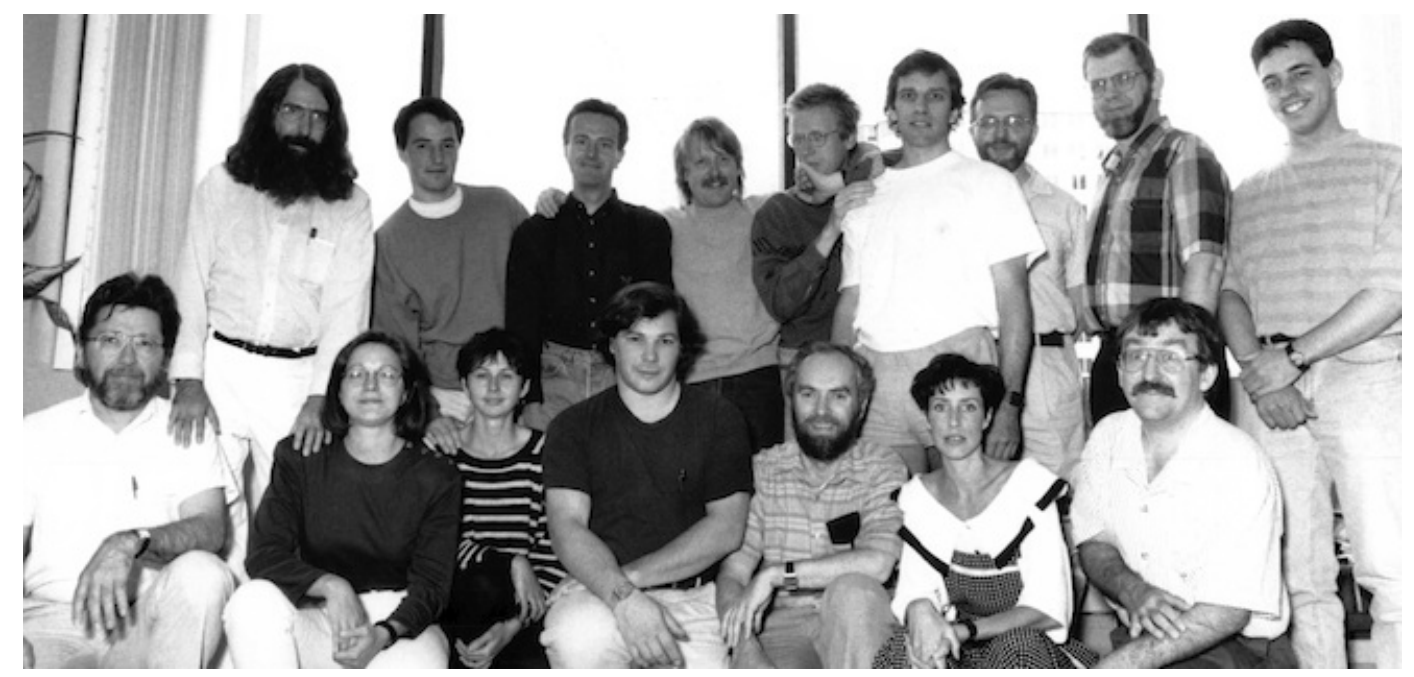

Figure 1: Some early members of the Groupe de recherche en combinatoire, LaCIM, UQÀM, around 1990. Bottom row: André Joyal, Louise Laforest, Odile Sheehy, François Bergeron, Pierre Leroux, Manon Blais, Jacques Labelle.

Upper row: Gilbert Labelle, Guillaume Cartier, Pierre Fortier, Srecko Brlek, Christophe Reutenauer, Alain Goupil, Pierre Bouchard, Timothy Walsh, Martin G. Ducharme.

At same time, inspired by the work of Dominique Foata on exponential generating series [23], André Joyal introduced the Theory of combinatorial species with his fundamental paper [33] (see also [34,35]). A species of structures is an endofunctor $F: \mathbf{B} \rightarrow \mathbf{B}$ of the category $\mathbf{B}$ of finite sets with bijections as morphisms. The following terminology is used : For each finite set $U$, an element $s$ of the corresponding set $F[U]$ is called an $F$-structure on (the underlying set) $U$ and for each bijection $f: U \rightarrow V$ between finite sets, the corresponding bijection $F[f]: F[U] \rightarrow F[V]$ is said to transport along $f$, each $F$-structure $s$ on $U$ to a corresponding $F$ structure $t=F[f](s)$ on $V$ (and $f$ is called an isomorphism from $s$ to $t$ ). For example, the species Gra of simple graphs is the functor Gra : B $\rightarrow$ B for which $\mathbf{G r a}[U]$ is the set of all simple graphs labelled by $U$ and $\mathbf{G r a}[f]$ transports each simple graph $s$ on $U$ to the corresponding isomorphic graph $t$ on $V$ along the graph isomorphism (relabelling) $f$. The main point is functoriality, since it requires that the transports must behave "coherently" : $F[g \circ f]=F[g] \circ F[f]$ whenever the composite bijection $g \circ f$ is defined. Because of functoriality, all the notions of labelled or unlabeled structures, automorphism groups of structures, etc, are automatically embodied within every species. Yeong-Nan-Yeh showed, in his Ph.D. Thesis under Stephen Schanuel [79] (see also [80]), that every species $F$ can be encoded by a very general formal series of the form $F=F(X)=\sum_{n, H} f_{n, H} X^{n} / H$ where, for each $n \geq 0, H$ runs through the subgroups of the symmetric group $S_{n}$ and $f_{n, H} \in \mathbb{N}$. Such series are called the molecular expansion of the species $F$.

We can associate to each species of structures symmetric functions as well as generating series and cycle index series for the enumeration of labeled or unlabeled structures in the spirit of Pólya theory. Several combinatorial 
operations - addition, multiplication (Cauchy as well as Hadamard), substitution, derivation, etc - allow us to build various species starting from other (generally simpler) species. These operations are compatible with corresponding operations on the above underlying formal series. This gives rise to combinatorial functional and differential equations facilitating the analysis of properties of the studied species of structures. The research group in combinatorics became in 1989, with Pierre Leroux as first director, an institutional research center at UQÀM called LaCIM: Laboratoire de Combinatoire et d'Informatique Mathématique (https://lacim.UQAM.ca/). The theory of species constitutes still today one of the main research axes of LaCIM. Many variants of the theory of species - linear species, relative species, many variables species, weighted species, virtual species (allowing minus signs), $\mathbb{K}$-species, tensorial species, $q$-species, Möbius species, cubical species, etc - have been created by members of LaCIM and various researchers around the world.

\section{His work on the Theory of Species and Combinatorics}

Let us now continue with the mathematical work of Pierre Leroux. He published in 1982, in collaboration with Hélène Décoste and Gilbert Labelle, an article on a combinatorial setting for the Newton-Raphson iteration [19]. In this paper, the root of the studied equation is the species of so-called "enriched" rooted trees [37], rather than a number, vector, or function as in numerical analysis. The Newton-Raphson iteration corresponds, in this context, to approximations of this species by producing rooted trees on increasingly large sets (whose cardinalities double at each step, reflecting the quadratic convergence in the context of classical analysis). Then come two fundamental articles, with Dominique Foata in 1983 [25] and Volker Strehl in 1985 [68] on combinatorial models of Jacobi polynomials and of various associated identities.

Then comes a series of papers (1985-91) [70-72] with Gérard Xavier Viennot, on the combinatorial resolution of systems of differential equations within the framework of linear-species, that is to say, species whose structures live on linearly ordered sets. This approach to differential equations provides, for example, a unified method for solving combinatorially differential problems of the type $y^{\prime}=f(x, y), y(0)=y_{0}$. In particular, the classical result of Désiré André who interprets, with the help alternating permutations, the coefficients of the power series solution $y=y(x)$ of the problem $y^{\prime}=1+y^{2}, y(0)=0$ follows immediately and perfectly automatically from this theory. Also, this approach to differential equations provides a new framework for iterated integrals in classical control theory $[31,60,73]$.

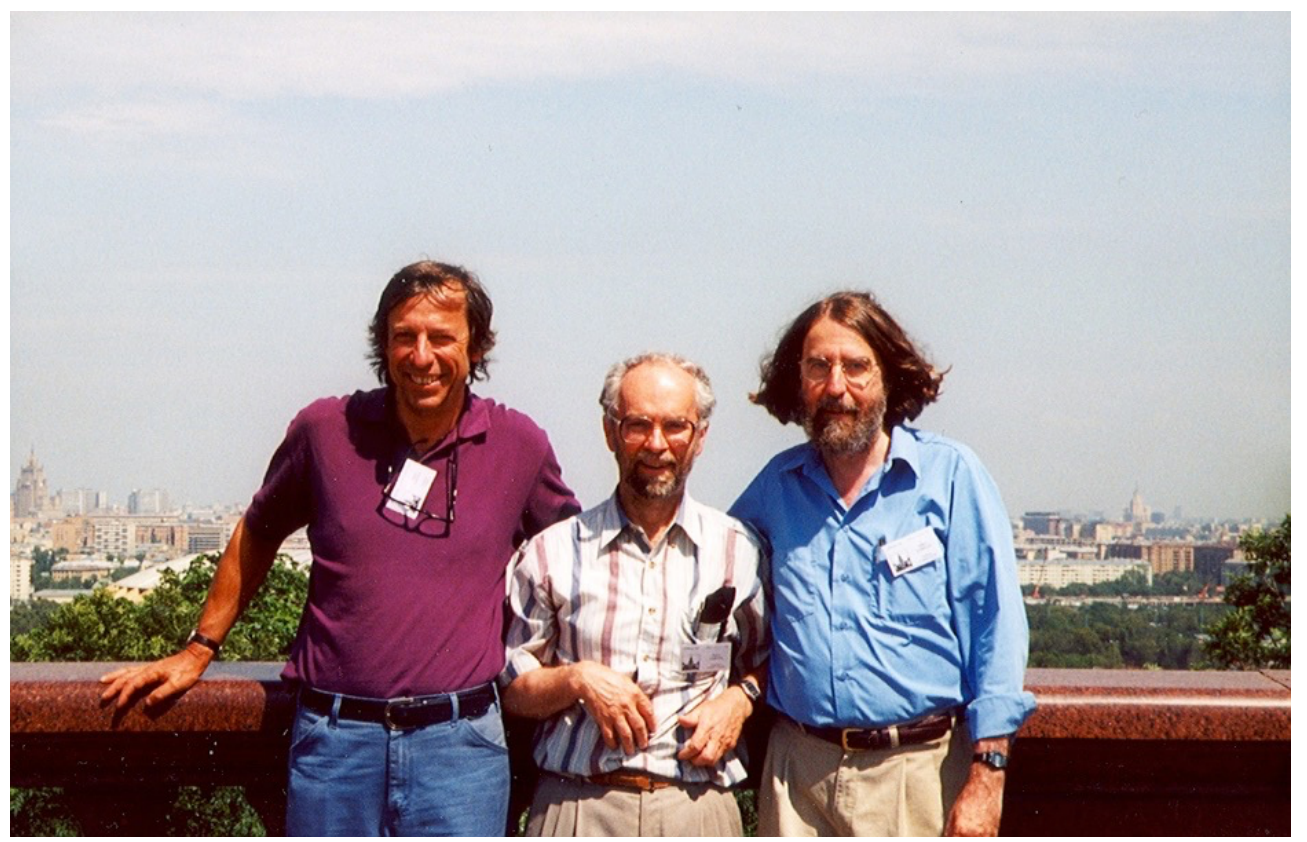

Figure 2: Gérard Xavier Viennot, Pierre Leroux, Gilbert Labelle, FPSAC 2000, Moscow.

Let us underline, in passing, the text Methoden der Anzahlbestimmung für einige Klassen von Graphen, Bayreuther Mathematische Schriften (1988) [56] in which Pierre describes the methods of the theory of species 
to the German mathematical community during a stay in Bayreuth.

After two articles on reduced matrices and the q-log convexity of q-Stirling numbers, in 1990 [57] and the $p, q$-Stirling numbers the same year with Anne de Medicis [16], Pierre Leroux continues with three articles on the theory of species: in 1991, with François Bergeron and Gilbert Labelle [9], on the expectation of the number of leaves of a tree having a given automorphism; in 1992, with Hélène Décoste and Gilbert Labelle [20] on the use of functorial composition (by opposition to partitional composition) in the theory of species and its applications to graph theory; in 1993, with Ibrahim Miloudi [61] on generalizations of Otter's formula in the form of combinatorial equations which connect families of trees (or graphs) to the same families when the structures are pointed. These equations, which he called dissymmetry formulas, allow us to elegantly analyze non-pointed structures by looking at the corresponding pointed ones having simpler automorphism groups and more easily characterized via combinatorial equations. In collaboration with Anne de Médicis in 1995, Leroux also published a text on generalized Stirling numbers, convolutions and corresponding p, $q$ analogues [17]. Note also his joint work with Étienne Rassart and Ariane Robitaille, Enumeration of Symmetry Classes of Convex Polyominoes in the Square Lattice [66], in which the authors enumerate and classify convex polyominoes according to their dihedral symmetry groups making use of various functional equations. This study is extended, with Étienne Rassart, to the class of parallelogram polyominoes in Enumeration of symmetry classes of Parallelogram Polyominoes [65]. Underline also the work Stirling Numbers Interpolation using Permutations with Forbidden Subsequences (2002) [49], made in collaboration with our Italian friends Elisa Pergola and Renzo Pinzani as well as Enumeration of Symmetry classes of Convex Polyominoes on the Honeycomb Lattice, (2005) [26] with Dominique Gouyou-Beauchamp. Pierre is one of the dozens of "checkers" of Doron Zeilberger's 1996 celebrated paper Proof of the Alternating Sign Matrix Conjecture [81].

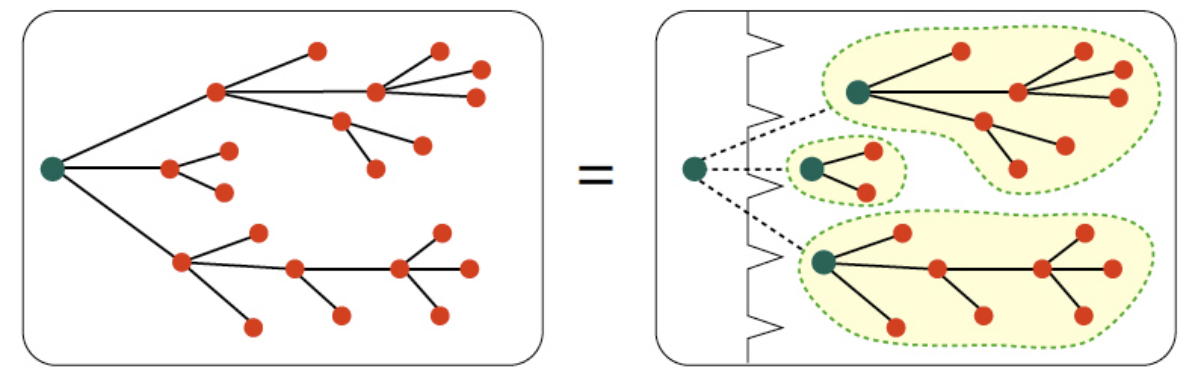

Figure 3: Rooted trees are singletons followed by a set of rooted trees: $T=X E(T)$.

From 1996, however, Pierre's work mostly focused on the theory of species and its applications. In $A n$ Extension of the Exponential Formula in Enumerative Combinatorics (1996) [42], in collaboration with Gilbert Labelle, a new virtual weighted species, noted $\Lambda^{(\alpha)}$, is introduced. It satisfies the remarkable property that it allows to add a weight counter, $\alpha$, for the connected components in the structures of any species $F$, satisfying $F(0)=1$, by forming another species, $F_{(\alpha)}$, via a simple substitution: $F_{(\alpha)}=\Lambda^{(\alpha)} \circ F_{+}$where $F_{+}=F-1$ is the species of $F$-structures on nonempty sets. For each such species $F$, this equation gives rise, canonically, to seven corresponding identities at the level of generating, $q$-generating, and some indicatrix series. The decomposition of the species $\Lambda^{(\alpha)}$, into homogeneous components constitutes a combinatorial extension of the binomial theorem of Newton for $(1+X)^{(\alpha)}$. Another co-author, Pierre Auger, then joined us and other combinatorial extensions of Newton's binomial and multinomial theorems appeared as expansions of the homogeneous components (according to cardinality of underlying sets or multisets) of any species of the form $M(1+X)$ or $M\left(X_{1}+\cdots+X_{m}\right)$, where $M=M(X)=X^{n} / H$ is a molecular species, $H$ being the stabilizer group of $M$-structures on $n$-element sets, see Generalized Binomial Coefficients for Molecular Species (2000) [3], and Combinatorial addition formulas and applications (2002) [4]. Also note the work Cubical Species and Nonassociative Algebras (1998) [32], with Gábor Heteyi, that introduces a variant of the theory species in which symmetric groups are replaced by hyperoctahedral groups, and the introduction, with Pierre Auger and Gilbert Labelle, of the Devmol package, written in the Maple computer algebra language, which calculates the expansion of the homogeneous components of an important class of species called cyclo-set species (i.e., the smallest class of species containing the species $C_{n}$ 
of $n$-cycles and the species $E_{n}$ of $n$-sets, $n=0,1,2, \ldots$, which is closed under the combinatorial operations of addition, multiplication, derivation, and substitution): Computing the molecular expansion of species with the Maple "Devmol" package (2003) [5].

In a series of papers, in collaboration with Miklos Bóna, Michel Bousquet, Cédric Chauve, Martin Ducharme, Tom Fowler, Ira Gessel, Gilbert Labelle, and Cédric Lamathe, several results concerning the classification and the exact or asymptotic enumeration of classes of trees, planar maps and non pointed cacti do their emergence through the introduction of appropriate dissymmetry formulas and functional equations: Enumeration of (unior bi-colored) plane trees according to their degree distribution (1996) [43], Enumeration of m-ary cacti (2000) [6], Enumeration of Planar two-face Map (2000) [13], The specification of 2-trees (2002) [24], Two bijective proofs of the arborescent form of the Good-Lagrange formula and some applications to colored rooted trees and cacti (2003) [12], A classification of plane and planar 2-trees (2003) [46], Labelled and unlabelled enumeration of $k$-gonal 2-trees (2004) [47], Enumeration of $k$-gonal 2-trees according to size and perimeter [48], A classification of plane trees (2006) [21].

Pierre Leroux is co-author with François Bergeron and Gilbert Labelle of the monograph Combinatorial species and tree-like structures [11] (see [10] for a French version, and Jacques Labelle [39] - [40] for an introduction to species) published by Cambridge University Press in 1998. In this 457 pages book, the authors make a synthesis of the work that had been done until then in the development of the theory of species by the Québec school LaCIM and other collaborators and researchers in the United States, France, and Germany, especially.

Pierre Leroux was the main organizer of the international Colloque de combinatoire énumérative, held at UQÀM (1985) whose proceedings are published in the Springer-Verlag Lecture Notes in Mathematics, no.1234 [41]. This historic congress brought together more than a hundred participants around the world, including a good number of renowned researchers in analytic and algebraic combinatorics*. It has contributed to the recognition of combinatorics as a real field of mathematics that has its own objectives, effective methods, and tools as well as very broad fields of application.
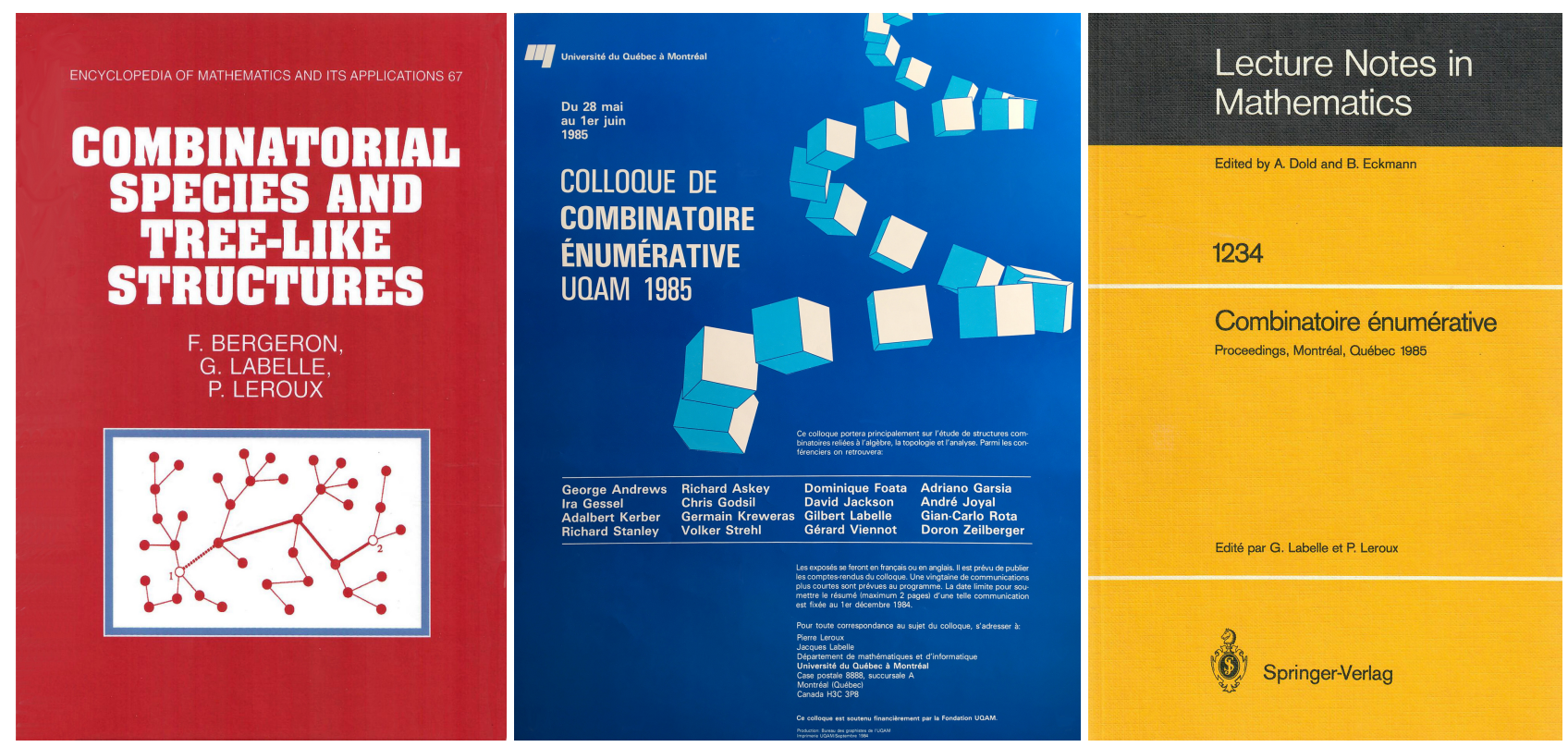

Figure 4: Book on species (Cambridge U. Press) [11], poster and Springer Lecture Notes vol. 1234 [41].

Here is an anecdote about these Proceedings: as co-editor with Pierre, it was I who opened, in his presence,

*Including (apart from local LaCIM members): Ashok K. Agarwal, George E. Andrews, Richard Askey, Anders Björner, David Bressoud, Neil J. Calkin, Luigi Cerlienco, Henry Crapo, Pierre Damphousse, Maylis Delest, Serge Dulucq, Omer Egecioglu, Dominique Foata, Adriano Garsia, Ira M. Gessel, Chris Godsil, Ian Goulden, Dominique Gouyou-Beauchamps, Curtis Greene, Werner Hässelbarth, David, J. Jackson, Gil Kalai, Adalbert Kerber, Germain Kreweras, Clement Lam, John McKay, Guy Melançon, Sri G. Mohanty, Tadepalli Naranaya, Oscar Nava, Heinrich Niederhausen, Kathy O'Hara, Joseph Oliveira, Peter Paule, Francesco Piras, Simon Plouffe, Robert W. Quackenbush, Don Rawlings, Eric Regener, Jeff Remmel, Bruce Richmond, Ivan Rival, Ivo Rosenberg, Gian-Carlo Rota, Ernst Ruch, Bruce Sagan, Richard Stanley, Dennis Stanton, Volker Strehl, Denis Thérien, Gabriel Thierrin, Loys Thimonier, Antonietta Venezia, Terry Visentin, Xavier G. Viennot, Dennis White, Yeong-Nan Yeh, Doron Zeilberger, Günter Ziegler. 
the letter from Springer-Verlag announcing the final acceptance of the monograph. To my surprise, I learned that Springer-Verlag had assigned the number 1234 to our Lecture Note. When I mentioned this information to Pierre, he immediately thought it was a fictitious bogus number and that the final number would come later. Upon closer examination, we realized that it was indeed number 1234 that Springer-Verlag had assigned us. What a beautiful coincidence for a book on enumerative combinatorics!

\section{Graph embeddings and statistical mechanics}

Another series of articles, with Andrei Gagarin and Gilbert Labelle, makes the bridge between the theory of species and the topology of embeddable graphs on surfaces such as the projective plane or the torus: Structure and labeled enumeration of $K_{33}$-subdivision-free Projective-Planar Graphs (2005) [27], Counting unlabelled toroidal graphs with no $K_{33}$-subdivisions (2007) [28] and The structure of $K_{33}$-subdivision-free toroidal graphs, (2007) [29]. In addition to classifying these species of graphs through functional equations and the use of a special substitution of classes of networks into the edges of graphs, the authors manage to enumerate these graphs up to isomorphism by switching to the Walsh indicatrix series which constitute a kind of extension of the indicatrix series of Pólya. In collaboration with Timothy Walsh, a new work, based on the previous ones deals with the study and the classification of 2-connected graphs whose 3-connected components are members of a given species of graphs: Two-connected graphs with prescribed three-connected components (2009) [30]. Pierre

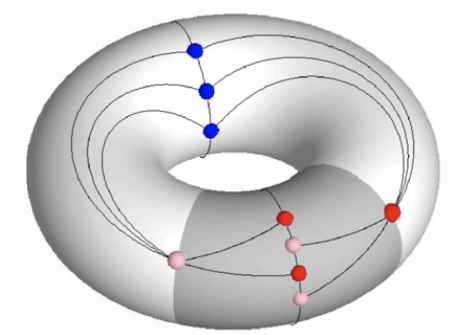

Figure 5: Graph embedded on a torus [29]

Leroux has also been interested in making connections between the theory of species and statistical mechanics. His first work on the subject is entitled Enumerative problems inspired by Mayers' theory of cluster integrals (2004) [59], followed, with the collaboration of Martin G. Ducharme and Gilbert Labelle, by Graph invariants arising from the theory of cluster integrals (2007) [22]. In these works, Mayer's integrals (cluster integrals) are related to the theory of species. Functional equations for species of graphs weighted by Mayer weights are established and efficient methods for calculating these weights are given for certain classes of graphs.

\section{Dissemination and promotion of research}

Pierre's mathematical career was also marked by his constant concern about the dissemination and promotion of research. He not only gave a multitude of mathematical conferences at national and international meetings but has also been an organizer of several important conferences. Apart from the UQÀM Colloquium SLN 1234 cited above, let us quote

- the Séminaire de combinatoire et d'informatique mathématique, at LaCIM, UQÀM (non-stop since 1979);

- the 3rd, 4th and 8th Formal Power series and Algebraic Combinatorics (SFCA/FPSAC), at LaBRI, Université Bordeaux I, (1991) [18], at LaCIM, UQÀM (1992) [63,64], at Minneapolis (1996) [69];

- the LaCIM 2000 Colloquium, at UQÀM [44,45];

- the School of Statistical Mechanics and combinatorics, Val-Morin, Québec (2007), organized with Mireille Bousquet-Mélou, Tony Guttmann, Alan Sokal [14]. 
- the workshop Combinatorial Identities and their Applications in Statistical Mechanics, Isaac Newton Institute for Mathematical Sciences, Cambridge, UK (2008) [1] (Pierre passed away just before the beginning of that Workshop for which he was co-organizer with Abdelmalek Abdesselam).

Note that from 1993 to 2002, he was a member of the permanent program committee (president from 1993 to 1998) of the annual SFCA/FPSAC international conferences. He was an invited professor at several institutions: Université de Nantes (1972); University of Calgary (1974); National Institute of Statistics and Applied Economics, Rabat, Morocco (1975); University of California, San Diego (1978-79); Université Bordeaux I (1984 and 2002); Universtät Erlangen-Nürnberg (1987); University of Minnesota (1987); University of Sidney (1994); University of Florence (2002); Université de Paris-Sud (2002).

From 1983 to 1986, he was administrative manager at UQÀM, of the review Topologie Structurale [76]. From 1988 to 1998, he was Chairman of the management committee of the international research journal Annales des sciences mathématiques du Québec [2]. He was also member of the editorial board of the Electronic Journal of Combinatorics (1993-2008) and Discrete Mathematics (1999-2008).

Finally, let us mention three texts by Pierre which were still under construction at the time of his death: Enumerating combinatorial structures equipped with a list of commuting automorphisms [75], with Miguel Mendez and Gilbert Labelle; Bridgeless graphs and the Legendre transform [8] with David Brydges; Mayer and ReeHoover weights of infinite families of 2-connected graphs [36] with his doctoral student Amel Kaouche.

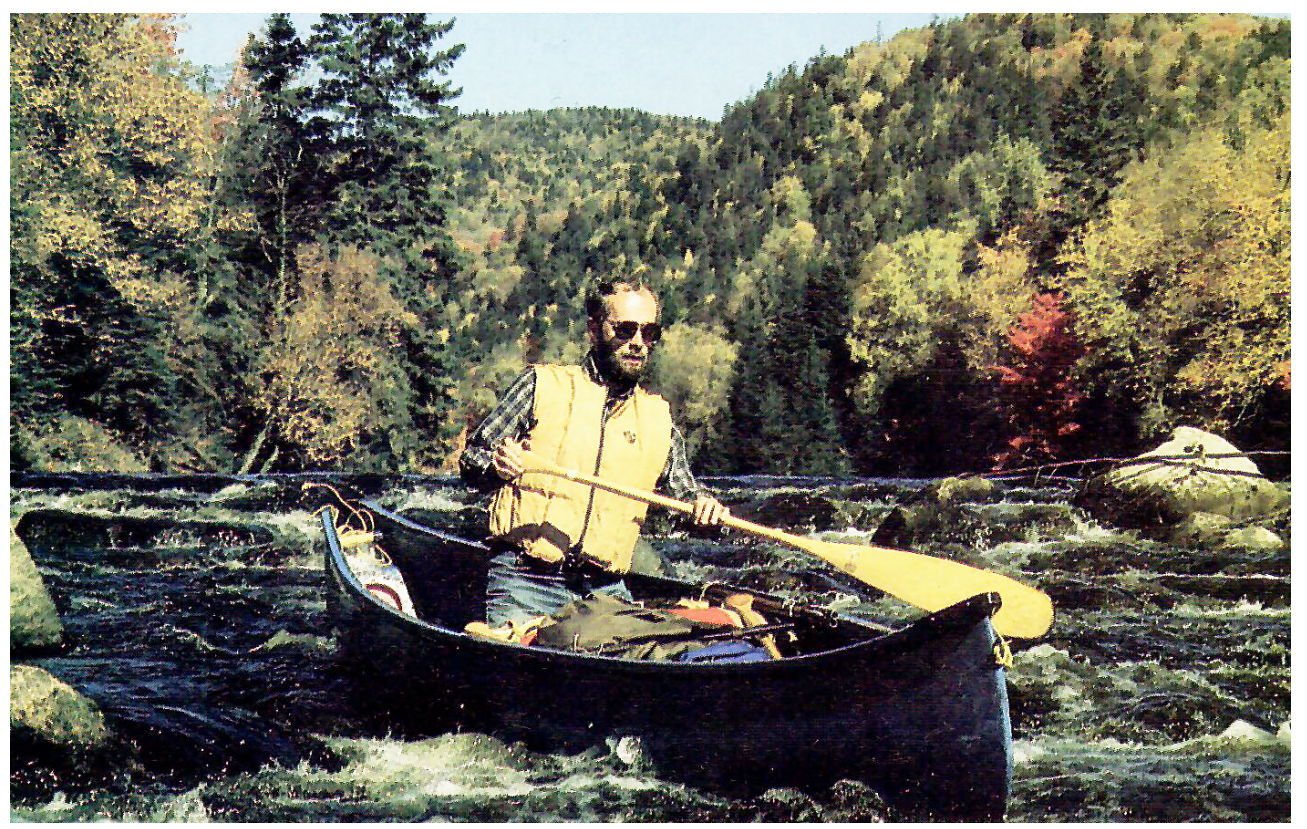

Figure 6: Pierre was among the founding members in 1969 of Canot Kayak Québec Federation

Pierre Leroux's dedication to others is legendary. To quote just a few examples among many others, let us mention that he was a supervisor for about forty master and doctoral students. He was a member of several internal committees at UQÀM. In particular, from 2004 to 2007, he was vice-dean for research at the Faculté des Sciences de l'UQÀM. From 2003 to 2007, he was co-president of a major development campaign of the Fondation de l'UQÀM. Two days before his death, he learned with great joy that the translation - in Braille of his well-known book [58] on linear algebra was just being completed ...

Xavier G. Viennot read a poem about Pierre, in French, at the beginning of a video-talk [78] he gave at Newton Institute in Cambridge, April 7 (2008), at the workshop "Combinatorial Identities and their Applications in Statistical Mechanics" cited above. This very nice poem is also available at the end of a paper he wrote about Pierre entitled Pierre Leroux (1942-2008) [77].

In 2011, during a ceremony bringing together his wife Madeleine Loubert, his two daughters Sophie and Marie-Claude, parents, and many friends, the UQÀM Faculté des Sciences awarded to Pierre Leroux the title Bâtisseur de la Recherche. 
Pierre was a great builder, an important mathematician, and a complete $\operatorname{man}^{\dagger}$.

\title{
BÂTISSEUR EN RECHERCHE 2011
}

Faculté des sciences

\section{Pierre Leroux}

Mettant à profit son esprit d'équipe, sa grande générosité intellectuelle et sa facilité à établir des contacts avec les meilleurs chercheurs du domaine, Pierre Leroux, professeur de mathématiques, a été I'instigateur d'un regroupement de chercheurs de haut niveau qui est devenu le centre institutionnel de recherche en combinatoire, le Lacim.

\section{UQĀM}

\author{
Juin 2011
}

Figure 7: Photo of a commemorative plaque affixed to the west wall of the Science Library, UQÀM

\section{References}

[1] A. Abdesselam and P. Leroux, Scientific organizers, Combinatorial Identities and their Applications in Statistical Mechanics, Workshop, Isaac Newton Institute for Mathematical Sciences, Cambridge, UK, April 7-11, 2008.

[2] Annales des sciences mathématiques du Québec. Centre de Recherches Mathématiques (CRM), Université de Montréal (1977-2012). Available at: http://www.labmath.uqam.ca/ annales/index.html

[3] P. Auger, G. Labelle, and P. Leroux, Generalized Binomial Coefficients for Molecular Species, J. Combin. Theory Ser. A 91 (2000), 15-48.

[4] P. Auger, G. Labelle, and P. Leroux, Combinatorial addition formulas and applications, Adv. in Appl. Math. 28 (2002), 302-342.

[5] P. Auger, G. Labelle, and P. Leroux, Computing the molecular expansion of species with the Maple package "Devmol", Sém. Lothar. Comin. 49 (2003), Article B49z.

[6] M. Bóna, M. Bousquet, G. Labelle, and P. Leroux, Enumeration of m-ary cacti, Adv. in Appl. Math. 24 (2000), 22-56.

[7] R.E. Block and P. Leroux, Generalized dual coalgebras of algebras, with applications to cofree coalgebras, J. Pure Appl. Algebra 36 (1985), 15-21.

[8] D. Brydges and P. Leroux, Bridgeless graphs and the Legendre transform, unpublished, 2008.

[9] F. Bergeron, G. Labelle, and P. Leroux, Computation of the expected number of leaves in a tree having a given automorphism, and related topics, Discrete Appl. Math. 34 (1991), 49-66.

[10] F. Bergeron, G. Labelle, and P. Leroux, Théorie des espèces et combinatoire des structures arborescentes, Publications du LACIM, Volume 19, 1994.

[11] F. Bergeron, G. Labelle, and P. Leroux, Combinatorial Species and Tree-like Structures, Monograph in the series Encyclopedia of Mathematics and its Applications, Volume 67, Cambridge University Press, 1998.

[12] M. Bousquet, C. Chauve, G. Labelle, and P. Leroux, Two bijective proofs of the arborescent form of the Good-Lagrange formula and some applications to colored rooted trees and cacti, Theoret. Comput. Sci. 307 (2003), 277-302.

\footnotetext{
${ }^{\dagger}$ For complementary information about Pierre Leroux see https://lacim.uqam.ca/membres-reguliers
} 
[13] M. Bousquet, G. Labelle, and P. Leroux, Enumeration of planar two-face maps, Discrete Math. 222 (2000), $1-25$.

[14] M. Bousquet-Mélou, T. Guttmann, P. Leroux, and A. Sokal (scientific organizers), School Statistical Mechanics and Combinatorics (C.R.M. Theme semester in recent advances in combinatorics), Far Hill Inn, Val-Morin (Québec) Canada, February 12-16, 2007.

[15] M. Content, F. Lemay, and P. Leroux, Catégorie de Möbius et fonctorialité, un cadre général pour l'inversion de Möbius, J. Combin. Theory Ser. A 28 (1980), 169-190.

[16] A. de Médicis and P. Leroux, A unified combinatorial approach for $q$ and p,q-Stirling numbers, Conference on Lattice Paths Combinatorics and Applications, McMaster University, Hamilton, 1990. J. Statist. Plann. Inference 34 (1993), 89-105.

[17] A. de Médicis and P. Leroux, Generalized Stirling Numbers, Convolution Formulae and p,q-analogues, Canad. J. Math. 47 (1995), 474-499.

[18] M. Delest, G. Jacob and P. Leroux, éditeurs, Séries formelles et combinatoire algébrique, Actes du 3e colloque, LaBRI, Bordeaux, May 2-4, 1991.

[19] H. Décoste, G. Labelle, and P. Leroux, Une approche combinatoire pour l'itération de Newton-Raphson, Adv. in Appl. Math. 3 (1982), 407-416.

[20] H. Décoste, G. Labelle, and P. Leroux, The functorial composition of species, a forgotten operation, Discrete Math. 99 (1992), 31-48.

[21] M. Ducharme, C. Lamathe, and P. Leroux, Une classifcation des arbres plans, Ann. Sci. Math. Québec $31: 2$ (2007), 139-154.

[22] M. G. Ducharme, G. Labelle, and P. Leroux, Graph weights arising from Mayers' theory of cluster integrals, Sém. Lothar. Comin. 54 (2007), Article B54m.

[23] D. Foata, La série génératrice exponentielle dans les problèmes d'énumération, Montréal, Presses de l'Université de Montréal, 1974.

[24] T. Fowler, I. Gessel, G. Labelle, and P. Leroux, The specification of 2-trees, Adv. in Appl. Math. 28 (2002), $145-168$.

[25] D. Foata and P. Leroux, Polynômes de Jacobi: interprétation combinatoire et fonction génératrice, Proc. Amer. Math. Soc. 87 (1983), 47-53.

[26] D. Gouyou-Beauchamp and P. Leroux, Enumeration of symmetry classes of convex polyominoes on the honeycomb lattice, Theoret. Comput. Sci. 346 (2005), 307-334.

[27] A. Gagarin, G. Labelle, and P. Leroux, Structure and labelled enumeration of $K_{33}$-subdivision-free projective-planar graphs, Pure Math. Appl. (PU.M.A.) 16:3 (2005), 267-286.

[28] A. Gagarin, G. Labelle, and P. Leroux, Counting unlabelled toroidal graphs with no K33-subdivisions, Adv. in Appl. Math. 39 (2007), 51-75.

[29] A. Gagarin, G. Labelle, and P. Leroux, The structure of $K_{33}$-subdivision-free toroidal graphs, Discrete Math. 307 (2007) 2993-3005.

[30] A. Gagarin, G. Labelle, P. Leroux, and T. Walsh, Two-connected graphs with prescribed three-connected components, Adv. in Appl. Math. 43 (2009), 46-74.

[31] C. Hespel, G. Jacob, F. Lamnabhi-Lagarrigue, P. Leroux and X. G. Viennot, Algebraic combinatorics and syntactic techniques in nonlinear control, in Proc. 3rd FPSAC, eds M. Delest, G. Jacob and P. Leroux, Bordeaux, 1991, p.245-261.

[32] G. Hetyei, G. Labelle, and P. Leroux, Cubical species and nonassociative algebras, Adv. in Appl. Math. 21 (1998), 499-546.

[33] A. Joyal, Une théorie combinatoire des séries formelles, Adv. Math. 42 (1981), 1-82.

[34] A. Joyal, Règle des signes en algèbre combinatoire, C. R. Acad. Sci. Paris Sér. I Math. du Canada VII, 5 (1985), 285-290. 
[35] A. Joyal, Foncteurs analytiques et espèces de structures, in: G. Labelle et P. Leroux, éds., Proceedings, Combinatoire énumérative, Montréal, Québec 1985, Lecture Notes in Mathematics 1234, Springer, Berlin, 1986, 126-159.

[36] A. Kaouche and P. Leroux, Mayer and Ree-Hoover weights of infinite families of 2-connected graphs, Sém. Lothar. Comin. 61A (2009), Article B61Af (presentation by A. Kaouche).

[37] G. Labelle, Une nouvelle démonstration des formules d'inversion de Lagrange, Adv. Math. $42: 3$ (1981), $217-241$.

[38] G. Labelle, La carrière mathématique de Pierre Leroux, Gaz. Math. 117 (2008), 67-74.

[39] J. Labelle, Applications diverses de la théorie combinatoire des espèces de structures, Ann. Sci. Math. Québec 7:1 (1983), 59-94.

[40] J. Labelle, Quelques espèces sur les ensembles de petite cardinalité, Ann. Sci. Math. Québec 9:1 (1985), $31-58$.

[41] G. Labelle et P. Leroux, éditeurs, Combinatoire énumérative, Montréal, Québec 1985, Proceedings, Lecture Notes in Mathematics 1234, Springer-Verlag, Heidelberg, 1986.

[42] G. Labelle and P. Leroux, An extension of the exponential formula in enumerative combinatorics, Electron. J. Combin. 3 (1996), Article \#R12.

[43] G. Labelle and P. Leroux, Enumeration of (uni- or bi-colored) plane trees according to their degree distribution, Discrete Math. 157 (1996), 227-240.

[44] G. Labelle et P. Leroux, éditeurs, Colloque LaCIM 2000, Combinatoire, Informatique et Applications, Actes du Colloque LaCIM 2000, LACIM-UQAM, 7-10 Septembre 2000, Publications du LACIM 27.

[45] G. Labelle and P. Leroux, Guest Editors, Numéro spécial consacré à LaCIM 2000, Conference on Combinatorics, Computer Science, and applications, Discrete Math. 256:3 (2002), 523-858.

[46] G. Labelle, C. Lamathe, and P. Leroux, A classification of plane and planar 2-trees, Theoret. Comput. Sci. 307 (2003), 337-363.

[47] G. Labelle, C. Lamathe, and P. Leroux, Labelled and unlabelled enumeration of k-gonal 2-trees, J. Combin. Theory Ser. A 106 (2004), 193-219.

[48] G. Labelle, C. Lamathe, and P. Leroux, Dénombrement des 2-arbres k-gonaux selon la taille et le périmètre, Ann. Sci. Math. Québec 29 (2005), 215-236.

[49] G. Labelle, P. Leroux, E. Pergola, and R. Pinzani, Stirling Numbers Interpolation using Permutations with Forbidden Subsequences, Discrete Math. 246 (2002), 177-195.

[50] P. Leroux, L'existence des enveloppes injectives, Mémoire de maîtrise, Université de Montréal, 1965.

[51] P. Leroux, Extension à des catégories de morphismes d'une paire de foncteurs adjoints, Thèse de doctorat, Université de Montréal, 1970.

[52] P. Leroux, Sur les structures d'effacement, Math. Z. 121 (1971), 329-340.

[53] P. Leroux, Une caractérisation de la catégorie des groupes, Canad. Math. Bull. 15 (1972) 375-380.

[54] P. Leroux, Les catégories de Möbius, 2e Colloque sur l'algèbre des catégories (Amiens 1975), Cah. Topol. Géom. Différ. Catég. XVI (1975), 280-282.

[55] P. Leroux, The Isomorphism Problem for Incidence Algebras of Möbius Categories, Illinois J. Math. 26 (1982), 52-61.

[56] P. Leroux, Methoden der Anzahlbestimmung für einige Klassen von Graphen, Bayreuth. Math. Schr. 26 (1988), 1-36.

[57] P. Leroux, Reduced matrices and q-log concavity properties of q-Stirling numbers, J. Combin. Theory Ser. A 54 (1990), 64-84.

[58] P. Leroux, Algèbre linéaire, une approche matricielle, Modulo-Editeur, 1983, Huitième tirage, 2001.

[59] P. Leroux, Enumerative problems inspired by Mayers' theory of cluster integrals, Electron. J. Combin. 11 (2004), Article \#R32. 
[60] F. Lamnabhi-Lagarrigue, P. Leroux and X. G. Viennot, Combinatorial approximations of Volterra series by bilinear systems, in Analysis by controlled dynamical systems, eds. Bonnard, Bride, Gauthier and Kupka, Birkäuser, Boston, 1991.

[61] P. Leroux and B. Miloudi, Généralisations de la Formule d’Otter, Ann. Sci. Math. Québec 16 (1992), 53-80.

[62] P. Leroux et P. Ribenboim, Dérivations d'ordre supérieur dans les catégories semiadditives, Cahiers de topologie et géométrie différentielle X (1970), 437-465.

[63] P. Leroux and C. Reutenauer, éditeurs, Séries formelles et combinatoire algébrique, Actes du 4e colloque, LACIM-UQAM, 15-19, 1992, Publications du LACIM, no 11, 487p.

[64] P. Leroux and C. Reutenauer, Guest editors, Numéro spécial consacré aux comptes-rendus arbitrés du 4e colloque sur les séries formelles et la combinatoire algébrique SFCA/FPSAC tenu à Montréal (UQAM) en juin 1992, Discrete Math. 139:1-3 (1995), 1-490.

[65] P. Leroux and E. Rassart, Enumeration of symmetry classes of Parallelogram Polyominoes, Ann. Sci. Math. Québec 25 (2001), 71-90.

[66] P. Leroux, E. Rassart, A. Robitaille, Enumeration of Symmetry Classes of Convex Polyominoes in the Square Lattice, Adv. in Appl. Math. 21 (1998), 343-380.

[67] P. Leroux and J. Sarraillé, Structure of Incidence Algebras of Graphs, Comm. Algebra 9 (1981), $1479-1517$.

[68] P. Leroux and V. Strehl, Jacobi polynomials : Combinatorics of the basic identities, Discrete Math. 57 (1985), 167-187.

[69] P. Leroux and D. Stanton, Guest Editors, Numéro spécial consacré aux comptes-rendus arbitrés du 8e colloque SFCA/FPSAC, Minneapolis, 1996, Discrete Math. 210:1-3 (2000), 1-184.

[70] P. Leroux and G. X. Viennot, Combinatorial resolution of systems of differential equations, I: Ordinary differential equations in: Combinatoire énumérative, Montréal, Québec 1985, Proceedings, Lecture Notes in Mathematics 1234, Springer-Verlag, 1986, 210-245.

[71] P. Leroux and G. X. Viennot, Résolution combinatoire des systèmes d'équations différentielles, II : Calcul intégral combinatoire, Ann. Sci. Math. Québec 12 (1988), 233-253.

[72] P. Leroux and G. X. Viennot, Combinatorial resolution of systems of differential equations, IV: Separation of variables, Discrete Math. 72 (1988), 237-250.

[73] P. Leroux and G. X. Viennot, A combinatorial approach to nonlinear functional expansions: an introduction with an example, 27th IEEE Conf. on Decision and Control, Austin, Texas, 1988, p. 1314-1319 [this is a summary of the paper published in Theoretical Comp. Science, 1991].

[74] P. Leroux and X. G. Viennot, A combinatorial approach to non linear functional expansions : An introduction with an example, Theoret. Comput. Sci. 79 (1991), 179-193.

[75] M. Mendez, G. Labelle, and P. Leroux, Enumerating combinatorial structures equipped with a list of commuting automorphisms, unpublished, 2008.

[76] Topologie structurale, A journal of the Structural Topology Research Group at the Université de Montréal and UQÀM (1979-1997). Available at http://www.iri.upc.edu/people/ros/StructuralTopology/.

[77] X. Viennot, Pierre Leroux (1942-2008), Gaz. Math. 117 (2008), 59-66.

[78] X. Viennot, Introduction to the theory of heaps of pieces with applications to statistical mechanics and quantum gravity, video-talk at Combinatorial Identities and their Applications in Statistical Mechanics Workshop, Isaac Newton Institute for Mathematical Sciences, Cambridge, UK, April 7 (2008). Available at http://www.viennot.org/abjc2-lectures.html

[79] Y.-N. Yeh, On the combinatorial species of Joyal, Ph.D. Thesis, State University of New-York, 1985.

[80] Y.-N. Yeh, The calculus of virtual species and $\mathbb{K}$-species, in: G. Labelle et P. Leroux, eds., Combinatoire énumérative, Montréal, Québec 1985, Proceedings, Lecture Notes in Mathematics, Volume 1234, Springer, Berlin, 1986, 351-369.

[81] D. Zeilberger (and dozens of "checkers", including Pierre Leroux), Proof of the Alternating Sign Matrix Conjecture, Electron. J. Combin. 3:2 (1996) (The Foata Festschrift volume), Article \#R13. 